\title{
Some Significant Advances in Spacer Fabric Technology for Newer Areas of Applications
}

\author{
Narayan Gokarneshan* and Velumani K \\ Department of Textile Technology, Park College of Engineering and Tekhnology, Coimbatore, Tamil Nadu, India
}

\begin{abstract}
Knit spacer fabrics have gained popularity in varied functional applications. Some important types of these fabrics are highlighted. One such is in medical applications, wherein the weft knit spacer fabrics have been attempted in treating pressure ulcer. The results show that the air permeability, thermal conductivity and water vapor permeability of weft knitted spacer fabrics are comparable with the existing wound dressings. The compressional resistance and resilience of them are good for providing protection as wound dressing. The absorbency of them is somewhat better than some wound dressings; however, they are suitable for pressure ulcer wounds with no heavy extrude. Warp knit spacer fabrics offer a better option over polyurethane foam in car seats owing to their advantages such as better recovery to compression, thermal properties and breathability. Flat-knitted spacer fabrics offer a strong potential for complex shape preforms, which could be used to manufacture composites with reduced waste and shorter production times. This article focuses on the improved strength and stiffness of woven three-dimensional (3D) spacer fabrics used in fiber reinforced plastics. The spacer fabrics were manufactured using E-glass/polypropylene (GF/PP) hybrid yarns and are intended for lightweight engineering applications. Flat-knitted spacer fabrics offer a strong potential for complex shape preforms, which could be used to manufacture composites with reduced waste and shorter production times.
\end{abstract}

Keywords: Automotive; Spacer fabric; Compression; Thermal property; Pressure ulcer; Wound; Dressing

\section{Introduction}

A good deal of research has been done in the area of spacer fabrics. Spacer fabrics can be of woven, nonwoven, and knitted types. Of these the knit spacer fabrics are the most prominent and find many and varied applications in technical textiles. When considering medical applications, pressure ulcer is one such. Pressure ulcer is the damage of skin or underlying tissue caused by prolonged pressure or pressure integrated with sheer and friction over a bony prominence which heel is one of the most common sites for pressure ulcer development [1-5]. In a new product development, designers must pay increased attention to environmental issues. In the motorcar industry, materials recycling have become a very important requirement. Fabrics and carpets inside cars are often composed of several layers of different materials, usually a polyester fabric laminated to a soft polyurethane foam backing by an adhesive. This type of construction makes disassembling virtually impossible, and the combination of different polymer chemistries for the fabric and backing makes recycling of the assembly extremely difficult. Furthermore, there is effluent emission from the flame bonding process, which is used to combine the different layers. As a result, the use of polyurethane foam in car interiors is environmentally hazardous both in terms of production and recycling [6]. Owing to their excellent mechanical properties, woven fabrics have numerous applications in the field of technical textiles. Some of their outstanding mechanical properties include high stiffness, strength, and dimensional stability. With the weaving process, dense woven structures with a load-compatible yarn orientation composed of high-performance materials (e.g., glass, carbon, aramide, polyethylentherephthalate) can be achieved, which in turn provide the basis for function- integrated, near-net shaped, and stiff spacer fabrics to be used as semi-finished products for fiber-reinforced plastics (FRPs) in lightweight engineering applications [7]. Spacer fabrics are complex three-dimensional (3D) constructions made of two separate fabric layers connected vertically with pile yarns or fabric layers. The conventional spacer fabrics composed of two surface layers bound with pile yarns are generally manufactured using weaving and knitting technologies. However, due to inferior mechanical properties, such as elasticity and deformability under applied loads, conventional spacer fabrics are not suitable for high-performance composite applications. Moreover, the restricted distance between the plane layers contributes to the drawbacks of such spacer fabrics. One solution is to connect the planes by means of vertical fabric layers instead of pile yarns. This type of $3 \mathrm{D}$ spacer fabric with multi-layer reinforcements in the fabric structures is expected to show superior mechanical properties and be especially suitable as textile preforms for lightweight composite applications [8-21].

\section{Weft Knit Spacer Fabrics for Treatment of Pressure Ulcers}

Despite the existence of wound dressings over the years, the management of wound is assuming greater complexities [22,23]. The modern wound dressings are designed to enhance wound healing [2426]. Moreover, the prevention and treatment of pressure sores need a significant amount of time and care. This not only greatly affects the lives of patients and their caretakers, but also the hospital services and costs of government as prolonged and expensive hospitalizations are required. Previous research has proven that the health costs of pressure ulcers are undoubtedly high $[3,27,28]$. It is difficult for pressure ulcers to heal and wound dressings that provide both good absorption and a cushioning effect are rare.

*Corresponding author: Narayan Gokarneshan, Professor and Head, Department of Textile Technology, Park College of Engineering and Tekhnology, Coimbatore, Tamil Nadu, India, Tel: +91-892626783; E-mail: advaitcbe@rediffmail.com

Received January 22, 2018; Accepted January 27, 2018; Published February 05, 2018

Citation: Gokarneshan N, Velumani K (2018) Some Significant Advances in Spacer Fabric Technology for Newer Areas of Applications. J Textile Sci Eng 8: 339. doi: 10.4172/2165-8064.1000339

Copyright: (c) 2018 Gokarneshan N, et al. This is an open-access article distributed under the terms of the Creative Commons Attribution License, which permits unrestricted use, distribution, and reproduction in any medium, provided the original author and source are credited. 
Spacer fabrics is a kind of three-dimensional knitted fabrics composited of top and bottom fabric with filament yarn in between to connect them together by tuck loop stitching $[1,29,30]$. Recently, weft knitted spacer fabric has gained great attention due to their versatile physical properties [31]. Based on the previous research, physical properties of spacer fabrics can be easily adjusted by adopting various types of spacer yarn, fabric density, thickness and fabric structure $[32,33]$. Therefore, it has been proven to have a wide area of applicability, including in medical products, due to their versatile physical, mechanical and thermal properties [34-36]. Their excellent ventilation and cushioning properties are also important for pressure ulcer prevention and the healing process [37]. The required physical properties of the absorbent layer of wound dressings, including air permeability, thermal conductivity, water permeability, absorbency and compression, are examined in 3-dimensional weft knitted spacer fabrics, and then evaluated and compared with those of wound dressings from the market. It aims to have a deeper understanding of the particular physical properties of weft knitted spacer fabrics tailored for use as the modern wound dressings.

The critical and necessary requirements of wound dressings for pressure ulcer are considered. Being a good wound dressing for pressure ulcer, not only require good absorbency property, but also breathability, thermal regulation and cushioning properties [37]. Therefore, the air permeability, thermal conductivity, water vapor permeability, absorbency and compression of weft knitted spacer fabrics are investigated and compared with existing wound dressings in order to evaluate whether the former can be used as a substitute for the latter. Based on the results, it proves that both air permeability and water vapour permeability of spacer fabrics are much better than that of wound dressings due to their structure. Although the thermal conductivity of spacer fabrics is not as good as all dressings, they are still comparable with one group of dressings. This is also true for the absorbency performance. Their absorbency property is comparable with another group of dressings. The results also prove that both compression resistance and compression resilience of some weft knitted spacer fabrics are better than that of wound dressings.

\section{Warp Knit Spacer Fabrics for Automotive Uses}

Besides ecological problems, car seats of present day should satisfy many needs. The car seats should not only appear attractive but should also very good mechanical properties with regard to durability and be able to protect passengers in the event of accident. Car seats must be comfortable too. This comfort must include both the mechanical support that the seat gives to the body as well as good climatic conditions, which are paramount for a driver's performance. Climatic comfort means good thermoregulation, which can balance the body's energy and offer good microclimate around the human skin. Studies have been conducted to measure the driver's rectal temperature with different types of car seats. With car seats offering good thermoregulation, after several hours of driving, the subject can still stay in the comfort region, i.e., below $37.5^{\circ} \mathrm{C}$ even in warm temperatures $\left(25^{\circ} \mathrm{C}\right)$.

On the other hand, some types of seats, which offer poor climatic comfort, will make the driver feel uncomfortably warm very quickly, as the rectal temperature rises to $37.5^{\circ} \mathrm{C}$ in approximately $40 \mathrm{~min}$. After $2 \mathrm{~h}$, the rectal temperature may rise to $38.2^{\circ} \mathrm{C}$, which is the limit of endurable strain for a normal person. Driving in such an uncomfortable seat, the driver must have a break; otherwise, he may have an accident caused by impaired physical and mental stress [38]. In recent years, the use of technical textiles has grown very fast. These fibrous materials, which have a variety of technical end uses, may also substitute some conventional materials with advantage in specific applications [39]. Warp-knitted spacer fabrics belong to this class, and they are very interesting structures due to their unique three-dimensional (3D) configuration. Their thickness may vary between 2 and $60 \mathrm{~mm}$ with good compressibility and breathability. A standard spacer fabric consists of two independent fabrics connected by spacer yarns, which complete the whole assembly [40]. To sustain the space between the two independent fabrics and to obtain the required compressive properties in the thickness direction, monofilament yarns are normally used as the spacer yarn. The material used may be polyamide, polypropylene and especially polyester. Moreover, the two independent fabrics may be knitted into any kind of mesh or plain structures to get the required dimensional, mechanical and comfort properties. Spacer fabrics present good resilience to compression, high bulk with relatively lightweight and very good moisture permeability for thermoregulation [41]. In addition, spacer fabrics are good at pressure relief $[42,43]$.

Warp-knitted spacer fabrics have compression properties different from those of normal fabrics because of the spacer yarn. Warp-knitted spacer fabrics show very good linear elastic compressibility in the first compression phase, which is of great interest for seats. The limit of the first compression phase varies with the bending rigidity and structure of the spacer yarn and with the thickness of the fabric. In this context, it is easy to design adequate WK spacer fabrics with suitable compression characteristic for use in car seats. Furthermore, warp-knitted spacer fabrics are better at reducing peak pressure than polyurethane foam. Using thicker warp-knitted spacer fabrics with appropriate spacer yarn structure and material, pressure can be much reduced.

Since there are limits to the thickness of warp knitted spacer fabrics, several layers may be used to get higher thicknesses and increased pressure relief [44]. Car seats with cushions of the warp-knitted spacer fabric are better at reducing peak pressures than seat cushions from polyurethane foam. In this context, the former will make drivers feel more comfortable. Air permeability of warp-knitted spacer fabrics is much better than polyurethane foams. Air permeability depends on the structure of the warp-knitted spacer fabrics. Therefore, these fabrics are more breathable substrates for car seats than polyurethane foam ones. Warp-knitted spacer fabrics have higher thermal conductivity and lower thermal resistance than polyurethane foams. Therefore, warp-knitted spacer fabrics can transfer heat more effectively away from the driver's body and so these fabrics have better thermoregulation properties than polyurethane foams for warm climatic conditions. Warp knitted spacer fabrics have good and comparable stability on thickness with polyurethane foams. Generally speaking, car seat cushions made of warp knitted spacer fabrics can offer good mechanical support and physical comfort to the driver's body. These fabrics are stronger than polyurethane foams, can be used for longer periods of time and can even be reused with new seats covers. Therefore, these fabrics used for seats are easier to recycle than polyurethane foam ones.

\section{New Weaving Technology}

Typical spacer weaves (so-called pile weaves) are constructed of woven outer layers, which are connected by additional pile yarns. Due to the composition of the aforementioned yarns, they provide the end composite with sufficient stability and compression. However, the mechanical properties of the fabric depend directly on the properties of the pile yarns, which have a limited bending stiffness. Pile weaves cannot be used for complexly shaped and/or highly strained FRPs. Woven spacer fabrics are constructed of woven outer layers and 
woven cross links instead of pile yarns. Spacer fabrics can be applied in multiple fields, such as the air and space industry, automotive industry, and the transportation branch in the form of wall elements, floor panels, inner coverings, and insulation as components in structural, efficient, and lightweight FRPs. Material properties (such as the amount of the material, the type of fiber, and the fabric geometry) and process properties (such as the number of warp beams and the weft, as well as the warp density) must be optimally combined to achieve a FRP that meets the demands of the end component (i.e., tensile strength, compressive strength, and flexural stiffness). Innovative weaving technology enables, in one process step, the production of near-net spacer fabrics with excellent mechanical properties [45,46]. Readymade, knitting, and gluing technologies can be used as an alternative method to the manufacture of spacer fabrics $[47,48]$. Yet, woven spacer fabrics have several advantages: elimination of the ready-made process, full utilization of the high-performance fiber substance, strengthening in the z-direction without crimp, structural variety, and productivity (e.g., for 416 tex hybrid yarn, an E7 flat knitting machine has a thread density of 81 yarns per $10 \mathrm{~cm}$, compared to a weaving machine with 200 yards per $10 \mathrm{~cm}$ and per layer).

Investigations have been carried out on the developed spacer fabrics constructed of GF/PP hybrid yarn for lightweight engineering applications. Multi-layer woven fabrics with weft of varying densities have been produced and tested for mechanical properties to increase the strength of spacer fabrics. The results of the studies show that through the production of three-layer fabrics (without yarn crimping), a reduction of yarn damage is achieved, as compared to fabrics with yarn crimping [49]. The measured values prove that the mechanical properties of the composites (such as tensile strength, flexural stiffness, and compression properties) are improved by a reduction in yarn damage and the non-crimped layers of the fabric structures. Composites made with crimped yarns show better impact properties compared to composites from three-layer fabrics. These contribute an important contingent to the further development of FRPs for demanding and high-performance applications. Woven spacer fabrics offer a high productivity and the greatest flexibility in adapting their material structure to the applied loads. Thus, they are pre-destined for lightweight engineering designs demanding complex structures and a multi-material design with an optimized material mix.

\section{Flat Knitted Spacer Fabrics for Light Weight Composite Applications}

It is foreseen that composites produced from 3D multi-layer spacer fabrics can have end uses that relate to the replacement of conventional panel structures that are being used for aircraft, transport vehicles, marine applications and infrastructures, lift cabins, and ballistic protection for buildings and combat vehicles, etc. But, for development of these innovative 3D spacer fabrics, modern electronic flat-knitting machines appear to be the best solution, since they are capable of manufacturing 3D complex-shaped engineering structures. Unique technical features that allow rapid and complex production include individual needle selection capability, the presence of holding down sinkers, presser-foots, racking, transfer, adapted feeding devices combined with a computer-aided design (CAD) system, and modern programming installations. Furthermore, the flexibility of the knitting process in combination with the possibility of integration of reinforcement yarns into fabric structures is capturing the attention of many researchers [50-52]. Moreover, knitted performs exhibit greater drape-ability and higher impact resistance in composites as compared to the commonly used textile structures (e.g., braiding, weaving, unidirectional techniques). In previous work, we reported the analysis and the manufacturing of 3D spacer fabrics on the basis of surface and connecting layers, the development of $3 \mathrm{D}$ spacer fabrics possessing only the weft inlays and the development of multi-layered 3D spacer fabrics. The research work documented in this direction includes the basic production principles of some flat-knitted spacer fabrics without reinforcements and the theoretical presentation of knitted sandwich spacer fabrics. Nevertheless, the further developments of flat-knitted multi-layered 3D spacer fabrics including an increased number of reinforcement layers into all individual fabrics are essential for high mechanical performance in complex-shaped lightweight composite applications. The sensor networks realized into $3 \mathrm{D}$ spacer fabric structures by means of integrating the functional yarns during the single-step manufacturing of the multi-layered 3D spacer fabrics would be the break-through advancement in fully automated manufacturing of function-integrated textile performs and such sensor networks could be used to monitor the structural perfection of end products. However, fibers, because of their aniosotropic mechanical properties and lower chemical and environmental resistances, cannot be used alone in structural engineering applications. They are, therefore, embedded in matrix materials to form fibrous composites. Fabrication of textile composites depends on the chemical nature of the matrix materials, which are generally thermoset and thermoplastic polymers. Thermoplastic composites are comprised of at least one reinforcement material and a thermoplastic polymer as matrix [53]. There are potentially a number of advantages for these composites as compared to thermoset composites. Owing to their high fracture toughness, easy recycling, elongation, short processing time, various forming possibilities, weld-ability, low cost, and resistance to Medias and corrosion, they appear to be more promising for some industrial applications [54]. Conversely, thermoplastic composite manufacturing routes are two-stage processes: firstly, a precursor material is formed using high-performance fibers and thermoplastic polymers, and, finally, the component is formed into the final product by applying high pressure and temperature. Nevertheless, the commingled hybrid yarns consisting of homogeneously mixed reinforcement and matrix filaments are soft, flexible, drapeable, and are available at low cost, which makes them a forerunner for thermoplastic composite applications.

The reinforcement component of the hybrid yarn is generally highperformance fibers, such as glass, carbon, and aramid fibers. Glass fibers are used extensively due to low material cost and higher mechanical properties. The thermoplastic matrix is used to fix the reinforcement components in a defined order for better bearing of applied forces to ensure good adhesion between the fibers and matrix material and to develop low-cost products, especially for the automobile industries [55]. The use of glass filaments (GFs) and polypropylene (PP) filaments in hybrid yarn in a volume combination of $52 \%$ and $48 \%$, respectively, is reported to optimize the mechanical properties of textile reinforced thermoplastic composites. Based on these parameters, the commingled hybrid yarn composed of GFs and PP filaments is preferred for the development of 3D spacer fabrics. One goal of the research program 'Textile-reinforced composite components for function-integrating multimaterial design in complex lightweight applications' by the German Research Foundation (SFB 639) at the Technische Universita t Dresden, is to develop multilayered 3D spacer fabrics as complexshaped textile preforms by using the flat-knitting technique in combination with hybrid yarns for high-performance thermoplastic composite applications. In order to achieve this objective, multi-layer reinforced 3D spacer fabrics curved in different angles (in the warp direction) were produced using exemplar GF-PP filament hybrid yarns 
following the recently developed flat-knitting techniques. With these new manufacturing techniques, reinforcement yarns were integrated up to four layers (two warp and two weft yarns aligned as a multi-layer structure) as biaxial inlays into all surface and connecting layers of spacer fabrics. Functional yarns were also integrated successfully into spacer fabric structures in a single processing step using the innovative integration concept in order to create sensor networks for structural health monitoring of end products.

Innovative 3D spacer fabrics consisting of individual surface and connecting layers including also the single axis curvatures to the wale direction were manufactured successfully using GF-PP filament hybrid yarns by the innovative flat-knitting technologies. In order to increase the wall thickness to improve the mechanical performance of the composites, up to four reinforcement layers of the reinforcement yarns were integrated into all surface and connecting layers. With the purpose of monitoring the structural health of end products, the flat-knitting technology was developed further for the creation of sensor networks by means of innovative integration of functional yarns into 3D spacer fabric structures during the manufacturing of the multi-layered $3 \mathrm{D}$ spacer fabrics. The easy integration of a wide spectrum of materials into multi-layered knit structures proves the extreme flexibilities of the latest technologies developed in this research. These could be a breakthrough advancement in fully automated manufacturing of functionintegrated textile performs where such seamless integration of functional yarns into the fabric structures is executed simultaneously, together with forming the multi-layered 3D spacer fabrics. Beyond the promising application trend in the area of lightweight composite structures, the novel 3D spacer fabrics produced with innovative flatknitting technologies (by cost-effective single-stage manufacturing) can also potentially be used in different fields, for example, in textilereinforced concretes, architectural designs, energy sectors, protective textiles, industrial textiles, and geo-textiles.

\section{Conclusion}

The structure and properties of knit spacer fabrics can be tailored to suit required end use applications. It has been found that air and water permeability of knit spacer fabrics are much better than that of wound dressings due to their structure. Findings have revealed that compression resistance as well as compression resilience of some weft knitted spacer fabrics are better than those of wound dressings. Due to the presence of spacer yarn warp knit spacer fabrics differ in compression properties from normal fabrics. They exhibit very good linear elastic compressibility during the first stage of compression which renders them suitable for seats. Warp Knit spacer fabrics with suitable compression characteristic can be used in car seats. Moreover, they are better at reducing peak pressure than polyurethane foam. A reinforced spacer fabric made of individual surface layers and joined with connecting layers shows improved mechanical properties for lightweight applications, such as textile based sandwich preforms. The development of flat-knitted multi-layered innovative threedimensional (3D) spacer fabrics from hybrid yarns consisting of glass and polypropylene filaments have been reported. Moreover, for structural health monitoring of composites, sensor networks could be created into a 3D spacer fabric structure in a single processing step through innovative integration of functional yarns. The twodimensional (2D) woven fabrics used for the outer layers of 3D spacer fabrics were produced from GF/PP hybrid yarns with different yarn alignments and weft densities. Furthermore, the implemented hybrid yarns and the 2D composites constructed of multi-layer fabrics were tested for mechanical properties. The processing of the GF/PP hybrid yarn, as well as the geometry of the woven fabrics, is proven to strongly influence the mechanical properties of the end composites. Multi-layer fabrics can be used for manufacturing woven 3D spacer fabrics with minimal yarn damage and high mechanical properties. A reinforced spacer fabric made of individual surface layers and joined with connecting layers shows improved mechanical properties for lightweight applications, such as textile based sandwich preforms. The development of flat-knitted multi-layered innovative threedimensional (3D) spacer fabrics from hybrid yarns consisting of glass and polypropylene filaments have been reported. Moreover, for structural health monitoring of composites, sensor networks could be created into a $3 \mathrm{D}$ spacer fabric structure in a single processing step through innovative integration of functional yarns.

\section{References}

1. Tong SF, Yip J, Yick KL, Yuen MCW (2015) Effects of different heel angles in sleep mode on heel interface pressure in the elderly. Clinical Biomechanics 32: $229-235$

2. Margolis DJ, Bilker W, Knauss J, Baumgarten M, Strom BL (2002) The incidence and prevalence of pressure ulcers among elderly patients in general medical practice. Annals of Epidemiology 12: 321-325

3. Pearson A, Francis K, Hodgkinson B, Curry G (2000) Prevalence and treatment of pressure ulcers in northern New South Wales. Australian Journal of Rura Health 8: 103-110.

4. Sopher R, Nixon J, McGinnis E, Gefen A (2011) The influence of foot posture support stiffness, heel pad loading and tissue mechanical properties on biomechanical factors associated with a risk of heel ulceration. Journal of the Mechanical Behavior of Biomedical Materials 4: 572-582.

5. Lyder $\mathrm{CH}$ (2003) Pressure ulcer prevention and management. JAMA 289: 223-226.

6. Wilkens C (1993) Raschel knitted spacer fabrics. Kettenwirk-Praxis 27: 59-63.

7. Hu J (2008) 3D fibrous assemblies' properties, applications and modeling of three-dimensional textile structures. Woodhead Publishing, Cambridge, 2008.

8. Savci S, Curiskis JI, Pailthorpe M (2001) Knittability of glass fiber weft-knitted preforms for composites. Textil Res J 71: 15-21.

9. Vuure AWV, Ko FK, Beevers C (2003) Net-shape knitting for complex composite preforms. Textil Res J 73: 1-10.

10. Abounaim M, Hoffmann G, Diestel O, Cherif C (2009) Development of flat knitted spacer fabrics for composites using hybrid yarns and investigation of $2 \mathrm{D}$ mechanical properties. Textil Res J 79: 596-610.

11. Abounaim M, Hoffmann G, Diestel O, Cherif C (2009) 3D spacer fabric as sandwich structure by flat knitting for composites using hybrid yarn. In Proceedings of the Autex Conference, Izmir, Turkey, pp: 675-681.

12. Abounaim M, Hoffmann G, Diestel O, Cherif C (2008) Flat knitted spacer fabrics with hybrid yarns for composite materials. Melliand Textileberichte 3-4: E30-31.

13. Abounaim M, Diestel O, Hoffmann G, Cherif C (2011) High performance thermoplastic composite from flat knitted multi-layer textile preform using hybrid yarn. Composites Science and Technology 71: 511-519.

14. Abounaim M, Hoffmann G, Diestel O, Cherif C (2011) Thermoplastic composite from innovative flat knitted 3D multi-layer spacer fabric using hybrid yarn and the study of 2D mechanical properties. Composites Science and Technology 70: $363-370$

15. Abounaim M, Hoffmann G, Diestel O, Cherif C (2010) Thermoplastic composite from curvilinear 3D multi-layer spacer fabric. J Rein Plast Comp 29: 3554-3565.

16. Abounaim M (2011) Process development for the manufacturing of flat knitted innovative 3D spacer fabrics for high performance composite applications. PhD thesis, Department of Mechanical Engineering, Technische Universita" tDresden, 2011.

17. Hong H, Araujo M, Fangueiro R (1996) 3D technical fabrics. Knitting Int 1232 55-57.

18. Araujo MD, Hong H, Fangueiro R, Ciobanu O, Ciobanu L (2001) Developments in weft-knitting technical textiles. In: Proceedings of the 1st Autex Conference (TECHNITEX 2001), Portugal 1:253-262. 
Citation: Gokarneshan N, Velumani K (2018) Some Significant Advances in Spacer Fabric Technology for Newer Areas of Applications. J Textile Sci Eng 8: 339. doi: 10.4172/2165-8064.1000339

19. Abounaim Md (2006) Modeling of technical bindings and manufacturing of flat Knitted and woven "spacer fabrics" with hybrid (GF/PP) yarn as sandwich structure. Master's thesis No.-1310, Department of Mechanical Engineering, Technische Universita" t Dresden, Germany, 2006.

20. Ciobanu L (2001) SANDTEX-developments on knitted sandwich fabrics with complex shapes. In: Proceedings of the 1st Autex Conference (TECNITEX 2001), Portugal 1: 490-496

21. Unal A, Hoffmann G, Cherif C (2006) Development of weft knitted spacer fabrics for composite materials. Melliand Textileberichte 4: E49-50.

22. Weller C, Sussman G (2006) Wound dressings update. Journal of Pharmacy Practice and Research 34: 318-324.

23. Maklebust J, Sieggreen M (2001) Pressure ulcers: Guidelines for prevention and management, Lippincott Williams \& Wilkins, 2001.

24. Augustine RO, Kalarikkal NA, Thomas SA (2014) Role of wound dressings in the management of chronic and acute diabetic wounds. Diabetes Mellit Hum Health Care Holist Approach Diagn Treat 273-314.

25. Basal G, Ilgaz SA (2009) A functional fabric for press urelcer prevention. Textile Research Journal 79: 1415-1426.

26. Ostadabbas S, Yousefi R, Faezipour M, Nourani M, Pompeo M (2011) Pressure ulcer prevention: An efficient turning schedule for bed-bound patients. In Life Science Systems and Applications Workshop (LiSSA), pp: 159-162.

27. Thomas DR (2001) Prevention and treatment of pressure ulcers: what works? What doesn't? Cleveland Clinic Journal of Medicine 68: 704-707.

28. Bruer SM, Powell N, Smith G (2005) Three-dimensionally knit spacer fabrics: a review of production techniques and applications. Journal of Textile and Apparel, Technology and Management 4: 1-31.

29. Bagherzadeh R, Montazer M, Latifi M, Sheikhzadeh M, Sattari M (2007) Evaluation of comfort properties of polyester knitted spacer fabrics finished with water repellent and antimicrobial agents. Fibers and Polymers 8: 386-392.

30. Liu Y, Hu H (2011) Compression property and air permeability of weft knitted spacer fabrics. The Journal of the Textile Institute 102: 366-372.

31. Armakan DM, Roye A (2009) A study on the compression behavior of space fabrics designed for concrete applications. Fibers and Polymers 10: 116-123.

32. Yip J, Ng SP (2008) Study of three-dimensional spacer fabrics: Physical and mechanical properties. Journal of Materials Processing Technology 206: 359-364.

33. Bagherzadeh R, Gorji M, Latifi M, Payvandy P, Kong LX (2012) Evolution of moisture management behavior of highwicking $3 \mathrm{D}$ warp knitted spacer fabrics. Fibers and Polymers 13: 529-534

34. Yip J, Ng SP (2009) Study of three-dimensional spacer fabrics: molding properties for intimate apparel application. Journal of Materials Processing Technology 209: 58-62

35. Tong SF, Yip J, Yick KL, Yuen MCW (2015) Exploring use of warp-knitted spacer fabric as a substitute for the absorbent layer for advanced wound dressing. Textile Research Journal 85: 1258-1268.

36. Shuk Fan T, Joanne $Y$, Kit lun $Y$ (2016) The possibility of using weft knitted spacer fabric as the wound dressing for pressure ulcer. Proceedings of ISERD International Conference, Helsinki, Finland, 5th-6th September 2016.

37. Umbach KH (2000) Physiological comfort on car seats. Kettenwirk-Praxis 34 9-12.

38. Heide M (2000) Spacer fabric with specific protective characteristics. MelliandTextilberichte 6: E124-E 125.
39. Rothe D (2001) Warp knitted spacer fabric-design and application fields. Knitting Technol 4: 14-16.

40. Schware D, Mohring U, Bartels VT (2005) Development of textiles for or coverings and pads. Melliand-Textilberichte 6: E95-E96

41. Ye X, Fanguiro R, Hu H (2005) Behavior of spacer knitted fabrics used as cushions. In 4th Central European Conference, 7-9 September 2005, Liberec Czech Republic.

42. Ye X, Hu H, Feng $X$ (2005) An experimental investigation on the properties of the spacer knitted fabrics for pressure reduction. Res J Text Apparel 9: 52-57.

43. Ye X, Fangueiro R, Hu H, de Araujo M (2010) Application of warp-knitted spacer fabric in car seats. Journal of the Textile Institute 98: 337-344.

44. http://www.lange-ritter.de/downloads/LR-Katalog_Glasfaser.pdf.

45. Badawi SSAM (2007) Development of the weaving machine and 3D woven spacer fabric structures for lightweight composites materials. Dissertation. Dresden: Department of Mechanical Engineering, Technische Universita" Dresden, 2007.

46. Herzberg C, Rodel H, Zhao N, Waldmann M, Zoerner G (2009) Konfektionstechnische Fertigung textile 3D-Preforms fu" $r$ komplexe Leichtbauanwendungen, Technical textile clothing production of 3D preforms for complex lightweight applications]. In: Proceedings of the $12^{\text {th }}$ Textile Technology Conference, Chemnitz, Germany, 30 September-1 October 2009, 231-235.

47. Adil M, Gerald H, Chokri C (2011) Development of weaving technology for manufacturing three-dimensional spacer fabrics with high-performance yarns for thermoplastic composite applications: An analysis of two-dimensional mechanical properties. Textile Research Journal 81: 1354-1366.

48. Torun AR, Paul C, Hanusch J, Diestel O, Hoffmann G, et al. (2007) Reinforced weft knitted preforms and spacer fabrics as well as woven spacer fabrics made of commingled hybrid yarns for RP. In: Proceedings of the Techtexti Symposium, Frankfurt, Germany, 12-14 June 2007.

49. Cherif C, Rodel H, Hoffmannn G, Diestel O, Herzberg C, et al. (2009) Textile Verarbeitungstechnologien fu" $r$ hybridgarnbasierte komplexe Preformstrukturen (Textile manufacturing technologies for hybrid based complex preform structures). Kunststofftech, J Plast Tech 2: 103-129.

50. Collaborative Research Centre. SFB 639, 'Textile-reinforced Composite Components for Function-integrating Multi-material Design in Complex Lightweight Applications', Technische Universita“ t Dresden, Germany, (accessed 18 February 2011).

51. Alagirusamy R, Ogale $\vee(2004)$ Commingled and air jet textured hybrid yarns for thermoplastic composites. J Ind Textil 33: 223-243.

52. Fujita A, Maekawa Z, Hamada H (1993) Mechanical behavior and fracture mechanism of thermoplastic composites with commingling yarn. J Rein Plast Comp 12: 156-172.

53. Sun BZ, Hu H, Gu BH (2010) Responses of 3D biaxial spacer weft-knitted composite circular plate under impact loading (part i: unit-cell and elastoplastic constitutive model). J Textil Inst 101: 28-34.

54. Li JJ, Sun BZ, Hu H, Gu BH (2010) Responses of 3D biaxial spacer weft-knitted composite circular plate under impact loading (part ii: impact tests and FEM calculation). J Textil Inst 101: 35-45

55. Zhang MX, Sun BZ, Hu H, Gu BH (2009) Dynamic behavior of 3D biaxial spacer weft-knitted composite t-beam under transverse impact. Mech Adv Mater Struct 16: $356-370$ 